\section{Eisbären-Endo am Fangzahn}

Kalt erwischt hat es den Eisbären Lars, Vater des berühmt gewordenen Berliner Eisbären Knut, der seit einem Jahr in einem dänischen Zoo lebt: Das riesige Tier litt unter einer Entzündung und wurde gleich an zwei Fangzähnen endodontisch behandelt. Eine Schweizer Dentalfirma hat den Eingriff an dem Raubtier per Video dokumentiert.

Eine örtliche Betäubung reichte für die Wurzelkanalbehandlung bei Lars nicht aus. Der Bär wurde in Vollnarkose gelegt, damit die Tier- und Zahnärzte vernünftig arbeiten konnten. Zehn Helfer waren allein nötig, um das 400 Kilo schwere Pelztier auf den aus Europaletten improvisierten Operationstisch unter freiem Himmel zu hieven. Lars bekam eine XXXL-Watterolle zwischen die Zähne, und dann ging es los mit der bärenstarken Endo-Behandlung samt mobiler digitaler Radiographie, mit deren Hilfe der Wurzelkanal dargestellt werden konnte. Die besondere Herausforderung lag darin, dass selbst mit der längsten Spezialfeile nicht der komplette, extrem lange Kanal bei dem riesigen Patienten erreicht werden konnte. Eine zweite Öffnung wurde erforderlich. Doch letztlich verlief die Operation erfolgreich, der Kanal wurde gespült und gefüllt mit recht hohem Materialeinsatz, wie das Video zeigt (zu finden beim Internetkanal Youtube unter dem Stichwort Endo
Treatment Polar Bear). Ob der 22-jährige Eisbär so recht wusste, wie ihm geschehen ist, weiß niemand. Nach der Narkose wirkte er noch etwas benommen, aber wohlauf. Ob das Füllmaterial, das eigentlich auf menschliche Bedürfnisse ausgelegt ist, mit Lars' Fressgewohnheiten klarkommt, wird sich in den nächsten Monaten zeigen.

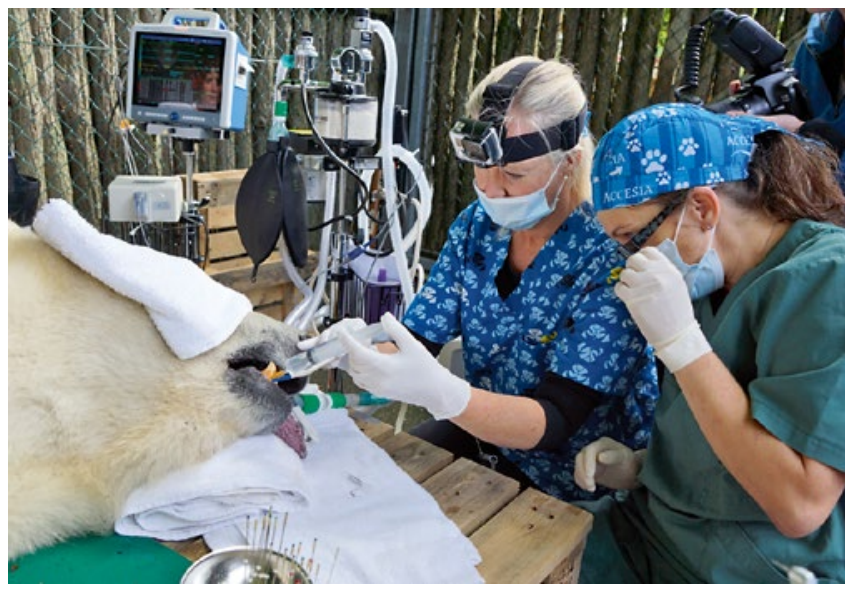

\title{
Statistik I
}

\section{Gesundheitsausgaben wachsen}

Die Ausgaben für Gesundheit steigen weiter: Nach Angaben des Statistischen Bundesamts (Destatis) lagen sie 2014 bei rund 328 Milliarden Euro. Das sind etwa 13 Milliarden Euro mehr als im Jahr zuvor. Insgesamt entspricht dies einer Pro-KopfAusgabe von knapp 4050 Euro. Nach Angaben von Destatis entfallen etwa 60 Prozent der Ausgaben (191 Milliarden Euro) auf die gesetzliche Krankenversicherung. Das entspricht rund zehn Milliarden Euro mehr als noch 2013. Die privaten Haushalte und privaten Organisationen schultern mit rund 43,2 Milliar- den Euro etwa 13 Prozent der Gesundheitsausgaben. Der Anteil der privaten Krankenversicherung bleibt wie im Vorjahr unverändert bei 29,3 Milliarden Euro (neun Prozent). Fast die Hälfte der Ausgaben floss der Statistik zufolge in den ambulanten Bereich (163,5 Milliarden Euro). Im Vergleich zu 2013 entspricht dies einem Anstieg um 5,2 Prozent. Nach Information des Statistischen Bundesamts gab es den stärksten Anstieg bei den ambulanten Pflegeinrichtungen (Plus 7,8 Prozent), auf die 13,3 Milliarden Euro entfielen.

\section{GKV-Finanzreserven schmelzen}

Bei den Rücklagen der gesetzlichen Krankenversicherung (GKV) hat sich der Negativtrend 2015 fortgesetzt. Wie das Bundesgesundheitsministerium (BMG) mitteilte, lagen die Reserven der Kassen und des Gesundheitsfonds zum Jahresende 2014 bei 24,5 Milliarden Euro. Ein Jahr zuvor verbuchte die GKV noch 28 Milliarden. Im Jahr 2013 lag das Polster sogar bei rund 30 Milliarden Euro. Laut BMG betrug der Anteil der gesetzlichen Kassen an den Reserven 14,5 Milliarden Euro. Die restlichen zehn Milliarden Euro steuerte der Gesundheitsfonds bei. Die gesetzlichen Krankenkassen verbuchten zum Jahresabschluss ein Defizit von 1,14 Milliarden Euro. Das BMG erklär- te das Defizit durch zu niedrige Zusatzbeiträge, die die Kassen aus Wettbewerbsgründen von den Versicherten verlangt hätten. Dadurch sei ein Minus von 900 Millionen Euro entstanden. Für Bundesgesundheitsminister Hermann Gröhe (CDU) sind die abnehmenden finanziellen Rücklagen der GKV kein Problem. „Mit Reserven von rund 24,5 Milliarden Euro steht die gesetzliche Krankenversicherung weiter auf einer stabilen Grundlage. Das ist auch das Ergebnis einer sorgfältig abwägenden Gesundheitspolitik, die Einnahmen und Ausgaben gleichermaßen im Blick behält", resümierte Gröhe in einer Pressemitteilung. 